

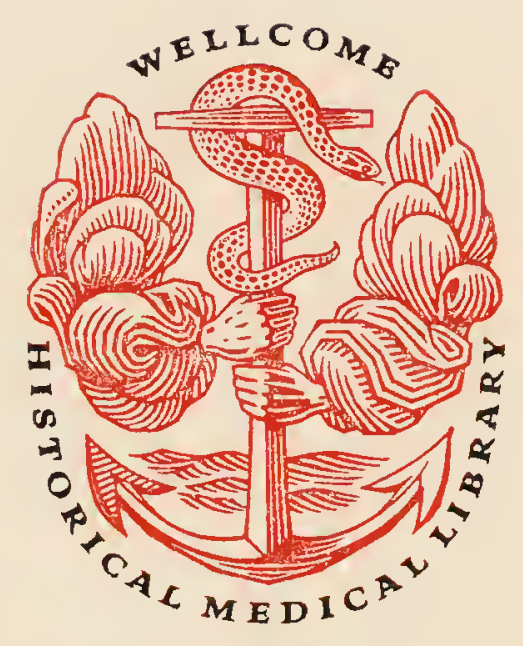

ACCESSION NUMBER

311090

PRESS MARK

$$
\text { SMITH, J.E. }
$$




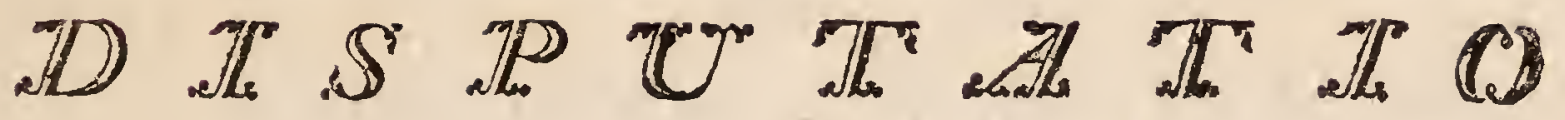

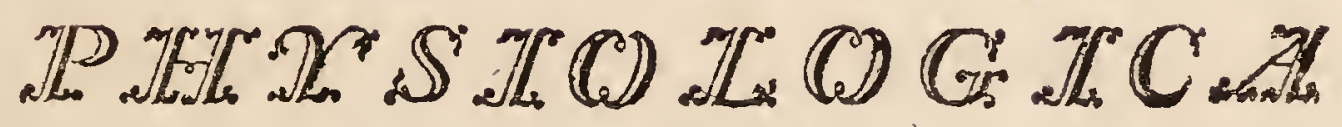

\author{
QUAEDAM
}

DE GENERATIONE COMPLECTENS。 


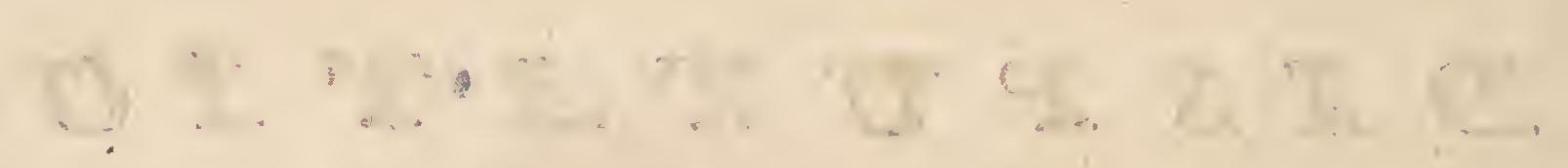

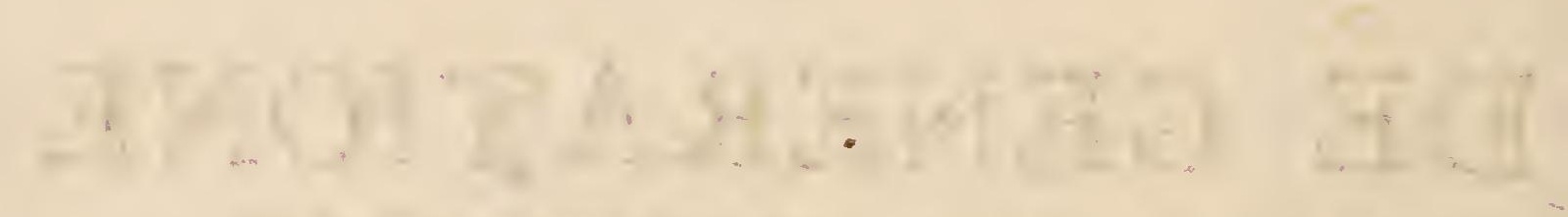

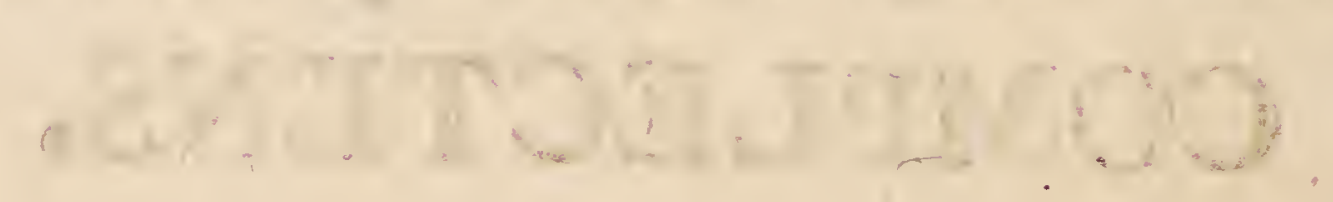




\section{DISPUTATIO PHTSIOLOGICA INAUGURALIS. \\ Q U A E D A M}

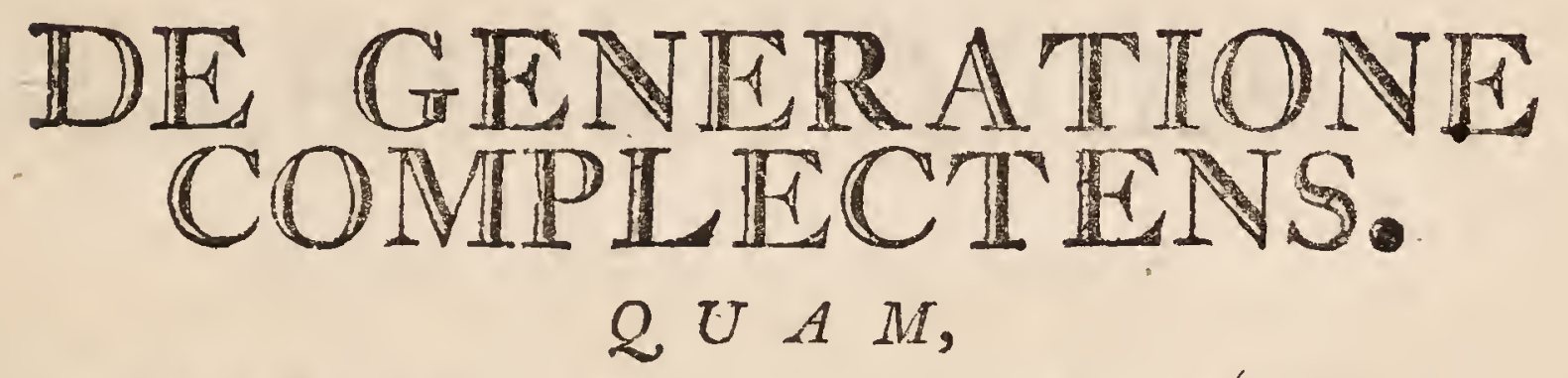

ANNUENTE SUMMO NUMINE, EX AUCTORITATERECTORIS MAGNIFICI,

\section{NICOLAI GEORGII OOSTERDYK, J O A N N. F I L.} MED.DOCT. MEDICINAE THEORETICAE ET PRACTICAE
PROFESSORIS ORDINARII; $\mathrm{N} E \mathrm{E} \quad \mathrm{N} Q \mathrm{~N}$ Amplifimi SENATUS ACADEMICI Consenfu, EO Nolilifinae FACULTATIS MEDICAE Dicreto,

PRO GRADLI DOCTORATUS,

Summisque in M E D I C IN A Honoribus \& Privilegiis rite ac legitime confequendis,

Eruditorum examini Jubmittit

F $A C O B U S$ EDUARDUS SMITH,

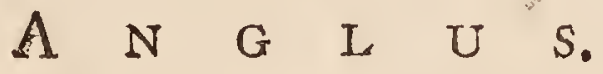

Soc. Reg. Lond. nec non Med Edin. Socius, Soc. Nat. Stud. Eilin. Praejes amnuus.

Ad diem xv. Jullii croroccl一xхvi H. L. Q. $S$.

$$
\text { LUGDUNI BATAVORUM, }
$$

$A P U D F R A R E S . M U R R A Y$,

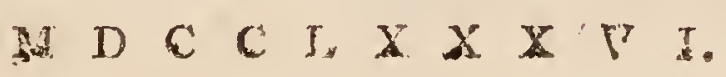





\section{SPECTANDIS VIRIS,}

\section{CONDISCIPULIS CARISSIMIS,}

\section{GULIELMOTHOMSON,}

MED. BACCALAUREO,

EX AEDE CHRISTI ALUMNO, \& PRAELECTORI ANATOMICES IN ACAD. OXONIENSI;

SOC. RLG. LOND. MED. ET NAT. STUD. PDIN. SOCIO: N E C N $\quad \mathrm{O} \quad \mathrm{N}$

\section{ROBERTO BATTY,}

E CORPORE CHIRURGORUM LONDINI SOC. MED. ET NAT. STUD. EDIN. SOCIO: Gratifimo functus munere,

Tentamen hoc physform (qua. le guale fit) offert in memoriam jiki

Jacobus Edus, South. 
Digitized by the Internet Archive . in 2018 with funding from Wellcome Library. 


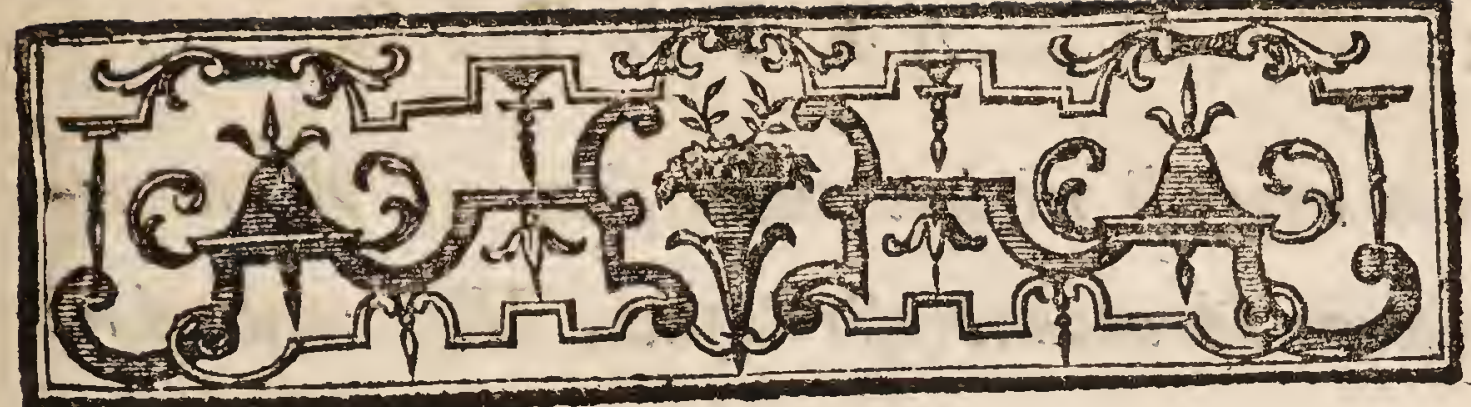

\section{$\mathbb{P} \mathbb{A} \mathbb{E} \mathbb{A} \mathbb{I}$.}

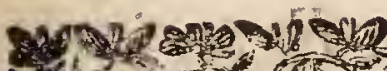

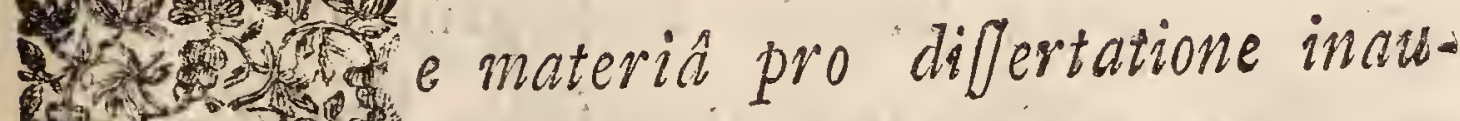
$D$ durali diu haerebam. Morbi fcilicet alicujus hiftoriam adumbra-

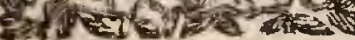
re yellem; non, ut Sacpe accidit, differtationem ex auctoribus practicis yel theoreticis, $\sqrt{i}$ ne ullâ propriâ experientiâ, componere; fed aliquid novi, quamis fortafe levis, offerre de morbi theoriâ yel medela: quaedam de catartho dicere, tum de aliis aliquibus phlogifficis, ut dicuntur, morbis, in animo fuife profiteor. Quum yero Syfematum hiforian patiobogicorum, Summamque illan obfcuritatenquae Scientian nedicam jam inde a majoribus obumbraret, animadieriffem, bujuccemodi disquigitio- 
nes aggreai minime aufus fum. Nullum ardorem ad arcana aftrologica, Hippocraie dice oruenda; neye ullum ad ineptias Galenicas de frigidis Galiais, $^{2}$ cocis humidifye ampleciendas fudium, in animo expertus fum. $\mathrm{Nik}$ gas theoreticas, aeternae optimi Sydenhamipaginae dedecora, hubenter deleyiffem. Experientiam, non totan yero theoriam, illuferiffmi Boerhaavii miratus Sum; neque profecto Lin. naeo s, Hypothefin Suam noyam de febrium intermittentium cause? invidi.

Vix magis me allexere recentifimorum theo. griae: nec inter omes auctores medicos inyemi caijus laureolas optarem, nific rariffmas itlas e naturae Sedulâ Scrutatione fidaque oberyatione acquifitas. Sedhaec conitici ornamenta yotis imperiti juvenis concedi yix Sperarem. Quid igitur milii reftat: Conatus quidem Sum de re generationis, prout ommibus aequaliter objeura, paucula quadam hinc inde fumpica 


\section{P R A E F T I O.}

proferre; ex quibus potius apparebit quantums ne $\int$ cimus quam quantum $\int c i m u s$ de mirabilibus naturae in corpore viventi arcanis.

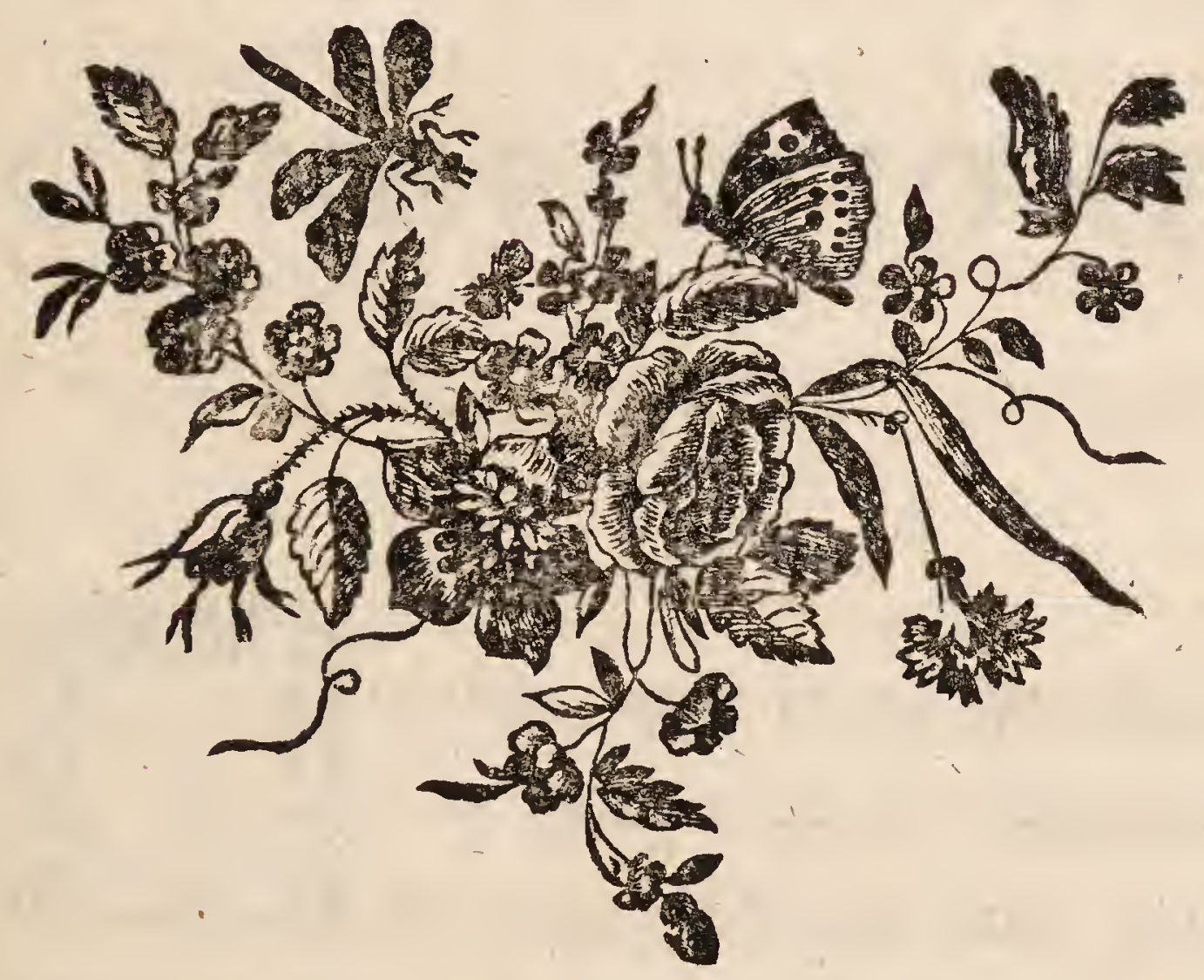


11

$$
.012 \mathrm{NAELS19}
$$

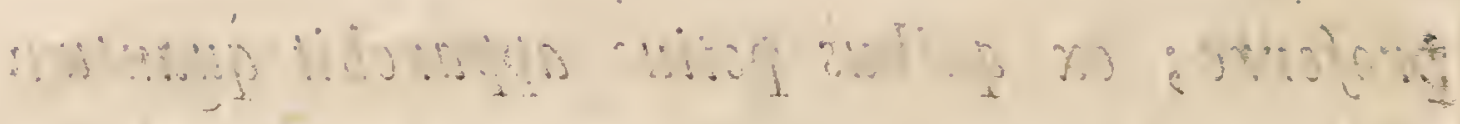

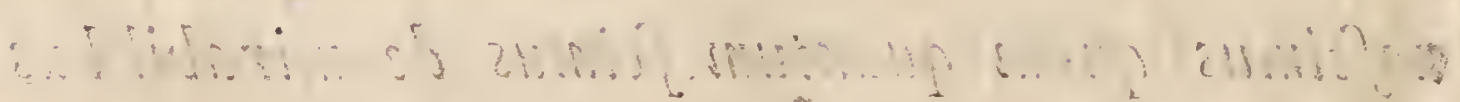

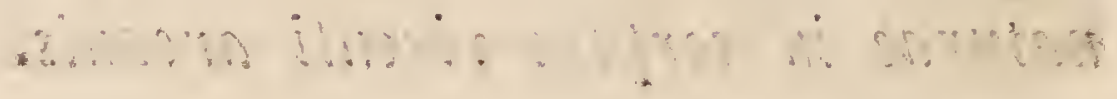

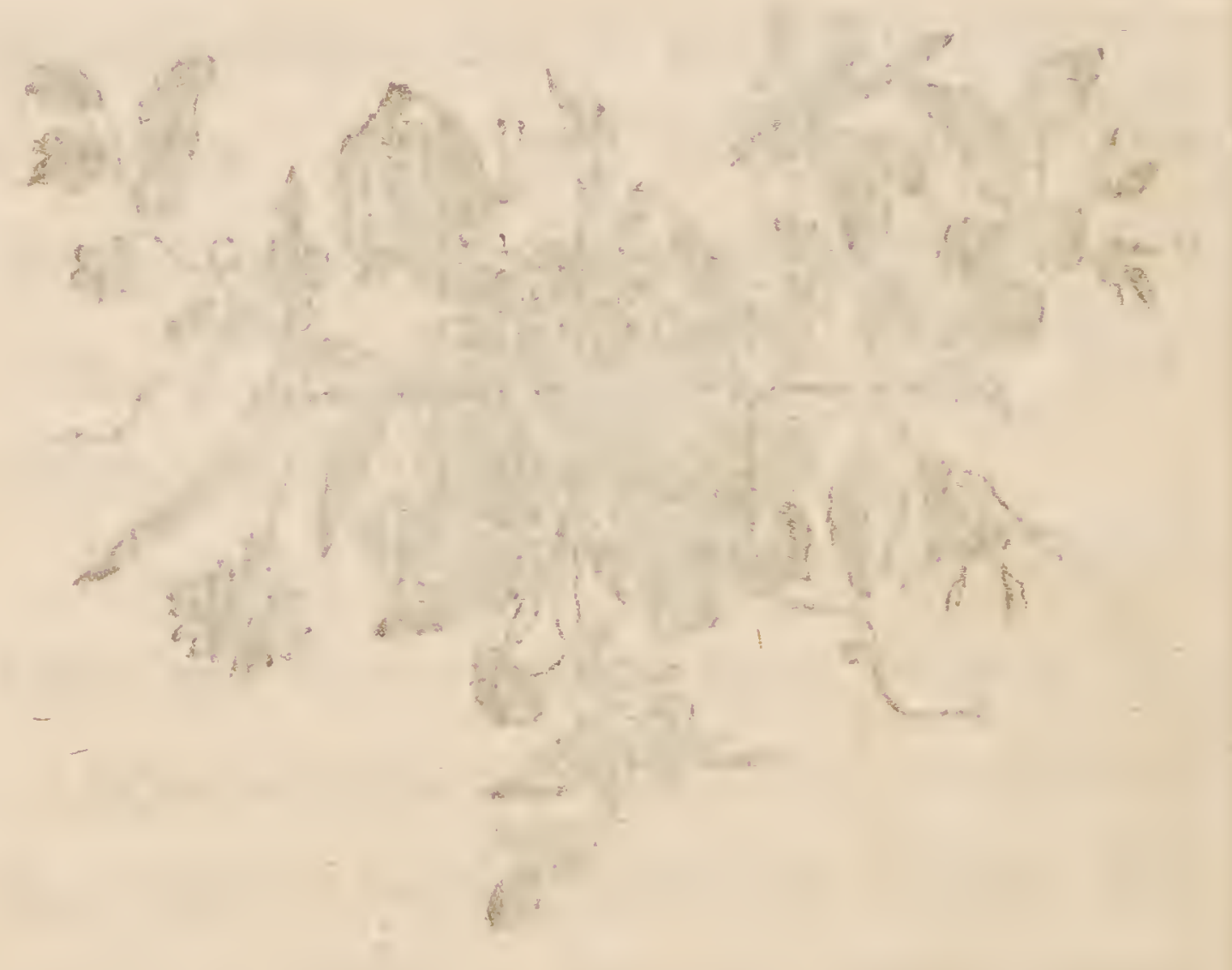




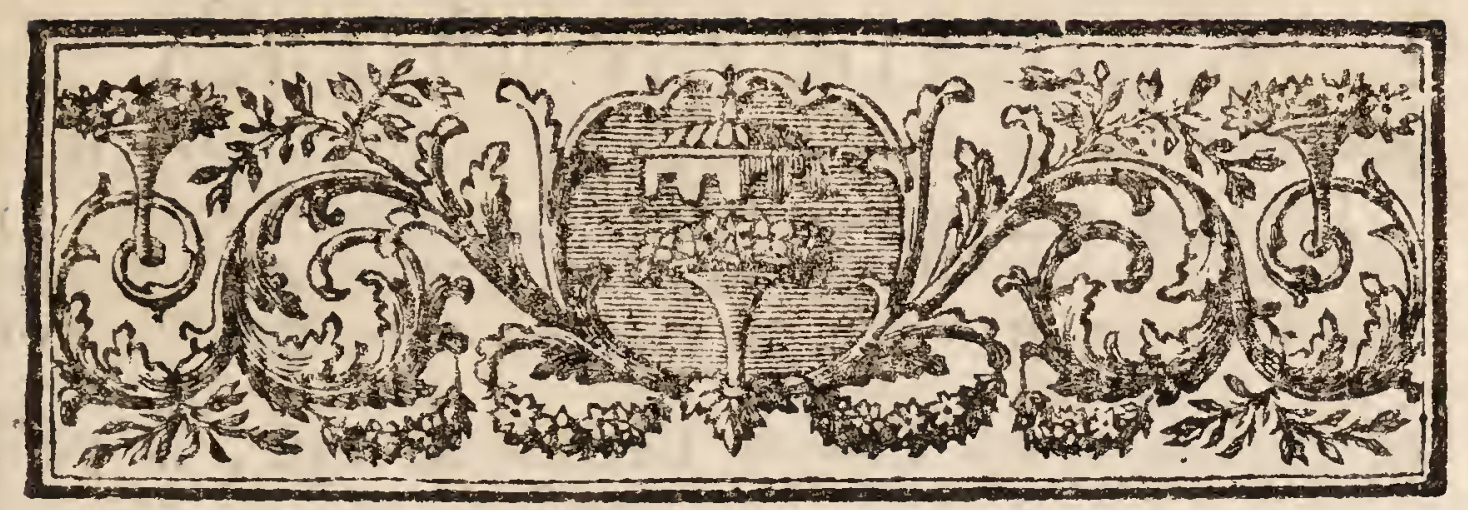

\title{
DISPUTATIO PHTSIOLOGICA
}

\author{
D. E
}

\section{GENERATIONE。}

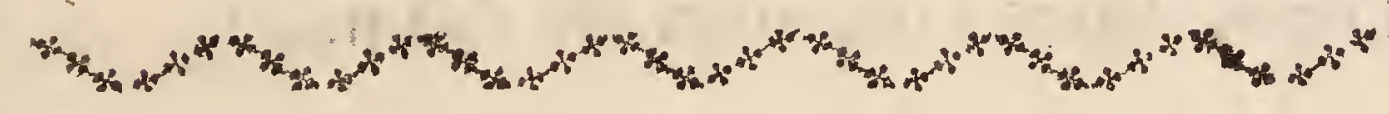

I.

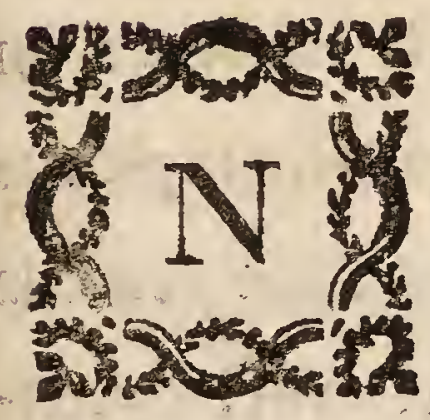

aturalia quae in tellure loceurrunt plerumque in tria Naturae regna diftribuuntur; Lapideum nempe, Vegetabile \& Animale.

Horm prius a duobus alteris facilline dignofcitur, organifacionis vitaeque defectu. 


\section{DISPUTATIO PHYSIOLOGICA}

Lapides crefcere feruntur. Accrefcunt tamen folummodo ex materiae ab externo depofitione, non ut Animalia vel Plantae nutrimenti ope, a vafis internis, magis vel minus compofitis, ad onnem partem delati.

\section{3.}

Ab animalibus primo intuitu, vegetabilia facillime dijudicanda exiftimentur ; anceps tamen haereo an vẹra fit difinctio.

„Vegetabilia crefcunt \& vivunt. Animalia cred "fcunt vivunt \& fentiunt", dixit Linnàets in Philofophiâ Botanica. Sed quomodo novimus plantas prorfus fenfucarere? Irritabilitati certiffme obnoxia funt, ut demonitrant Berbetis, Apocyni, Syngenefiarumque variarum famina, nec non Mimofá folia. Motum fpontaneum etiam plantis ineffe fuadent Parnafiac, Rutacque impregnatio, Valifne. riae phaenomenon ftupendum. Argumentum etiam non mediocre fumendum eft $\mathrm{cx}$ foliorum motu in Helyfaro gyranti. 


\section{GINERATIONE.}

Vegetabilia loco motivitate gaudere vix dici potest, neque omnia quidem animalia, Exemplum praebent Taenia in inteftinis fixa, Zoophyta va sia faxis radicata, quae fine ullo voluntario motu in aeternum quiefcerent, nifi aliquando haec un. dae, illam aftrictio relaxatioque inteftinorun, mo: tatione, non fuâ, uiti cogerent.

Defeetus ventriculi in plantis a quibusdam pro certiffimo difcrimine declaratur. Sed Taenia etiam ventriculo caret; aortam enim ex ore incipientem unice jactare docet Clariff. Mon ro in Praelectionibus anatomicis.

$$
4 .
$$

Vegetabilia, aeque ac Animalia, ope or: ganorum quae fexualia vocamus propagantur.

In animalibus perfectioribus dictis, quadripedibus nempe, avibusque, propagationem factam. effe per generationem folam notifimum eft: Ne-

$$
\text { A 2. que }
$$




\section{D:SPUTATIO PHYSIOLOGICA}

que hoc minus certe in infectis, pifcibus \& forfan amphibiis, accidit. Pari ratione dicimus quod omnes plantae floribus five organis generationis praeditae, horum ope propagantur. Et quoniam haec organa in omnibus vegetabilibus quae rite examinandi facultas conceffa fuit, reperimus, ex analogia colligimus, omnia vegetabilia fexuali ge: neratione propagari.

\section{5.}

Omnia fere Vegetabilia alio etian modo fefe propagandi gaudent, gemmis nempe e radice vel caule ortis, quae cafu vel arte a caudice parente feparatae, novam formant plantam, progreftu temporis generationi aptam.

$$
\sigma .
$$

Quaedam animalia e vermium ordine eo: dem modo propagantur. E corpore paren. tis, gemmae undique oriuntur, fponteque 


\section{DE GENERATIONE. 5}

ex illo difcedunt, \& perfecta fiunt animalcula.

\section{7.}

Cognitio generationis fexualis optime ex obfervatione animalium majorum, nec non plantarum floribus felicioribus praeditarum petenda.

\section{8.}

In utrisque bina funt organa quae hoc officio funguntur, quorum differentia fexus differentiam conftituunt.

\section{9.}

Faeminei fexus organa intus in fe fovent prolis rudimenta, adhuc inanimata; Mafculi autem materiem peculiarem producunt, à vi cujus ftimulanti \& nutrienti vita \& incrementum tenellis dantur embryonibus.
A 3
Vide 


\section{DISPUTATIO PHYSIOLOGICA}

Vide Halleri Meni oiresfurla formation du coeug dans le Poulet, Erc. „Bonnet fur les Corps Or: ganifes, Spalanzani Experimenta, \&c.

\section{IO.}

In Animalibus fpecies hermaphroditica vi occurrit; \& licet fubinde in eodem individuo organa mafcula \& faeminea inveniantur, vix tamen per fe raborollal conficere queant, fine amatore hermaphrodito.

Exemplum frifunt Helices plures vulgares; fed aliter in Aphidibus fe res habet.

Vide Bonnet fur les Corps Organifs.

II.

Vegetabilia e contririo plerumque hermaphrodita funt. Organa corum mafcula, quae famina antherifera dicuntur, plerumque in coden flore una cum organis foemineis (pi- 


\section{DE GENER ATIONE.
ftillis) collocantur; pittilla autem in fuo}

flore conclufa, pollen (five farina faecun. dans) ex antheris emiffum maxima ex parte faecundat. At non haec folum; nam per - aëra pollen libere vagum, non illis omnino proprium dicatur.

In multis vegetabilibus ftamina valde piftillis numero fuperant, ut in Capparide, Theâ \&xc; nec ullum cum feminum numero rationem habent, ex gr. in Tiliâ, Mammeâ, \&c. In aliis piftilla \& femina longe ftaminibus numerofiora funt, ut in Myofuro, ubi paucifima haec vix ad impreguationem tot faeminarum furfere videntur.

\section{2.}

Animalium pauca monogama funt, ut Homo; fed ptura polygama, ut Bos, Onis, ubi mas finguius ad impregnationem numerofiffimi faeminarum gregis fuficiat. Inter Vegetabilia vix exemplum verae monogamiae de $=$ 


\section{\& DISPUTATIO PHYSIOLOGICA}

deprehendi poteft, nifi forfitan in Hippuri. de, \& plantis fcitamineis monandris.

\section{3 .}

Neque actio pollinis vagi alicujus plantae, plantis ejusdem fpeciei impregnandis femper cohibita eft; hinc inter affines faepe partus hybridus, \& ex mente Linnaei plures, quas credimus fpecies diftinctas effe, hoc modo aliquando ortae funt.

Vide Linna Difquifitionem de fexu Plantarum; \& Difr. de Plantis Hybridis, Amoen. Acad. Vol. 3 , ubi Genera etian hybrida momorat illuftriffimus ille auctor.

Inter infecia etiam adulteria compicinntur, \& forte inter alia animalia confpici poffint. 


\section{UE GENERATIONE.}

Exempla reperienda inter Chrylomelas \& Coc: cinellas.

$$
15 .
$$

Pollen foecundans plantarum e granulis five capfulis conftat, quae quamdiu ficcae fuerint, inertia manent; fed fi forfan rorem piftilli attigerint, diffiliunt, \& vi elafticâ exhalationem prolificam per ftylum ad feminum rudimenta demittunt.

Pollen integrum non intrare per ftylum abunde probatur ex infpectione Amaryllidis formofiffimae. Tulipae, Mirabilis, nec non Hibifcorum, Malvarim, Liliorum, \&c.

$$
16 .
$$

Sic fluidum feminale animalium maximâ ex parte vehiculum folum effe videtur; nam quicquid emiffum fuerit, ab organis foemi- 


\section{Io DISPUTATIO PHYSIOLOGICA}

neis fere prorfus rejicitur, nec ovaria attin: gere poteft.

$$
1 \%
$$

Experimenta adhuc celebrata, praecipue chemica, ad naturam feminis animalium detegendam omnino fere inania videntur.

Inter haec maxime dubia funt illa celeberrim Comitis de Buffon, e veficularum feminalium liquore, qui ex parte tantum vehiculum feminis eft, inftituta.

$$
\text { i8. }
$$

Semen ex omnibus partibus corporis elaboratum vel elicitum effe, valde ridenda eft opinio.

Nec anatomiae nec legitinae phyfiologiae, mon. friferae autem theoriae hoc dogma tribuendumeft. dillis enim difcimus femen mafculinum e male 


\section{DE GENERATIONE. II}

fanguinis, aeque ac alios humores, fecerni. Quicquid enim ex illis collegimus de feminis origine vel phaenomenis, qro magis illud effe eiz toto corpore contractuni, vel ad generationem aptum, pari jure de urinâ, vel muco narium fauciumque affirmari poteft. Secernitur nempe e mo1e: fanguinis duabus parvis arteriis, quarum ora, a-corde longe remota pauxillulo tantum fanguinis obvia funt, \& faciem prae fe fert a muco non valde diverfam. Nil miror illam quam hodierni refpuimus de femine opinionem olim acceptam: fuiffe, cum circulatio fanguinis, vafa abforbentia, nervorum ufus \& natura, atque animati corporis ftructura (quae $a b$ internâ tranfudatione longiffime: abhorret) e tenebris nondum emerfiffent: fed quan. to fupore afficimur cum in hypothefe clarifimi Buffon hoc dogma de induftria memoratum leginius!

19.

Praefcripta qualiacunque ad liberos alteru: trius generis, five mafculi five foeminei, 


\section{I2 DISPUTATIO PHYSIOLOGICA}

pro arbitrio procreandos, omnino futilia \& improbanda funt.

Si, ut fupra propofuimus (fec, 9) organifatio foetus primaria a matre deriuetur; fi primae ejus lineae in ovario virgineo latentes, nihil nifi ftimus: lum feminis mafculini a quo animentur expec: tant; femen nihil ad fexum foetus determinaldum, (qui a conformatione illâ maternâ primaria pendet quâ embryones mafeuli a foemineis differunt, ) efficere valeat. Probabile eft ovarium fingulum ova aliquot cujusvis fexus continere, ue plantae monoicae in eodem individuo flores mafeulos \& foemineos prodicunt. Hinc omnino fortuitum effe oportet utrum mafculum an foemineum ovum ab halitu feminis impregnatum fit, nec ullo confilio aut arte halicus ifte ad hoc vel illud potệt dirigi.

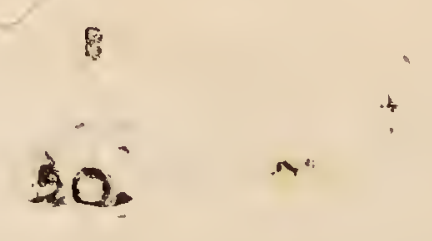

Stimulus vel actio femisis mafculini in o- 


\section{DE GENERATIONE}

vum minimè ad foetum maturandum fufficet, qui omnino, polt primam impregnationem, a viribus pendet maternis. Si plura fucrint ova impregnata, tot embryonum nutritionem fufcipit natura, quot hae vires ad perfectionem ducere valeant.

Hujus exempla quaedam in genere humano retulere auctores, fed minus certa; Vide Pouppe Defportes, "Hift. des Máladies de San Domingue".

In plantis exempla plurima reperienda; nullibi tammen manifeftiora quam in Coffêa arabicâ (in regione Yemen dictâ) Spontaneâ, cujus flores unicum tantum femen perficiunt, vix unquam duo, quamvis in altis rupibus, ventis ab onni parte expofita crefcat, loco foecundationi benigno, fed Colo fterilifimo. - Cautio haud abfimilis naturae videri poteft in Hordeo fativo, in campis areticis culto, nec non in Lathyro odorato, plantâ indicâ hortorum eưtopaeorum hodie gratiffimo ornamento; in utrisque flores ruperiores etiam ant impregnationem femper marcefcunt. 


\section{DISPUTATIO PHYSIOLOGICA}

$2 I$.

Aơtrs generationis magnâ cum excitatio: ne fyftematis nervofi comitatur, praecipue in animalibus perfectioribus; in ordinibus inferioribus, vermibus fcilicet, fi adfit, nos hactenus fefellit, neque in plantis ulla ejus irdicia videmus. Vis ejus nullam cum mole relativa cerebri, vix cum folertiâ animalis habet rationem.

Infocta quaedam, quibus nullum adeft cerebrum (quamvis magna tamen fagacitas) admodum falacia funt, adeo ut faepe ex uno actu mas omnino exhauritur \& miferrime perit, ut in Gryllo. Homo, quamvis omnium animalium maximâ pro corporis magnitudine cerebri mole praeditus, pluribus animalibus, quoad aefum venereum, videtur ce. dere. 
22.

Tabes dorfalis, cum aliis nimia venereae indulgentiae effectibus, ifti nervorum exci. tationi unice tribuenda.

Quantam operan \& oleum perdidere auctores qui antiquam iftam pathologiam humoralen amplexi funt, in explicando hoc phaenomeno! Hipo pocrates, vagae cujusdam theoriae caufa, quâ femen e medullâ fpinali de:ivari finxit, hunc morbum tabem dorfalem nominavit, \& fymptomata ejus ad injurias quasdam huic organo illatas, retulit. Sequentes auktores, divi fenis miro acumine practico induct, theorias ejus praepropere in fecuti funt. Hinc de deficcatione corporis, nec non de lumborum tabe, ad hure usque dicm aliquando audimus: deinde ortae funt hypothefes de femine ex omni quidern particulâ corporis fecrem to, qua ratione totum corpus ex illius profufion ne marcefcere facile credebatur. Pathologi tandem recentiores, \& inter cos optimus ille Tiffot, quam 


\section{I.6 DISPUTÁTIO PHYSIOLOGICA \&c.}

vis non prorfus verba Hippocratica rejecit, tabis ejus dorfalis caufam magis philofophice nee non magis fimpliciter explicare conatus eft, nempe ex magna ob coitum fyftematis nervofi irritatione: Ingeniofiffimus \& celeberrimus Johannes Hunter hanc opinionem ftrenue amplexus eft, \& etian affernit labefactionem corporis nullam oriri ex me. râ feminis effufione absque incitatione venereâ.

Hanc hypothefin de nimii coitûs operandi mo: do confirmar ejusdem cffectum praecipuorum contemplatio. Nervos omnes \& fenfûs organa, oculos fcilicet áurefque maxime debilitat. Irritabilitatem auget. Ventriculo, cujus fanitas a firmitate fyftematis nervofí quam maxime pendet, \& qui ex illius languore faepe mirifice turbatur, varias adfert affertiones morbofas. Dolores lancinantes capitis frequentes funt. Memoria minuitur, confunditur. Poftea fupervenit Syncope, Spasmi, Epileplia, Paralyfis, Caecitas, Mors.

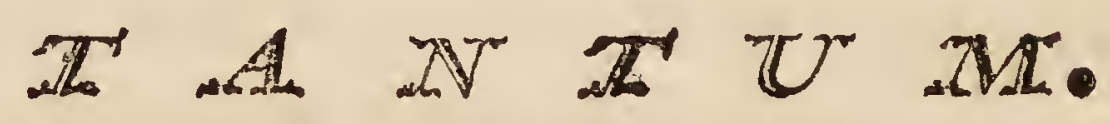




\section{$\mathbb{I} \mathbb{I} \mathbb{S} \mathbb{E}$.}

Qui hifforiam naturas, naturae etiom Creatorem ille colit.

Omnis motus yitalis in nervis infitus eft.

$$
3 \cdot
$$

Periofecum Samum vix fenfibilitate goudet.

$$
4 .
$$

In corpore viventi tranjudatio mulla efle poi teft.

$$
5 .
$$

In cadayere transudatio univer:falis efto

6.

Nomenclatur anatomic caftigatione quam maxime eget. 
Nec rubeola nec yariola femper phlogificus. morbus eft.

\section{8.}

Medicamentorum e regno animali paucifom utilia, e regno minerali pauca Eo periculoSa, e regna vegetabile egregia EO multifaric desumi polfunt.

\section{9.}

Phamacopaeis fore omnibus multa expungenda, multa etiom addenda, vidit Sana So docta - objeryatio.

IO.

Pharmacopolis botanices inciiis ne nimium medicus credeto.
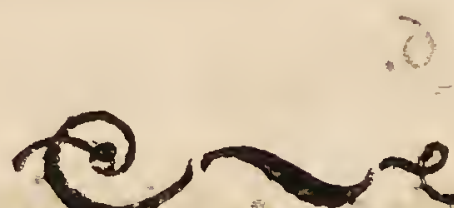

